\title{
Analysis of liquid-type proof mass under oscillating conditions
}

\author{
Dong-Joon Won ${ }^{1}$, Sangmin Lee $2^{2^{*}}$ and Joonwon Kim ${ }^{1^{*}}$ (D)
}

\begin{abstract}
In this study, the spring constant of an accelerometer with a liquid-type proof mass was analyzed. Unlike a general solid-type microelectromechanical system accelerometer, the Laplace pressure is considered a restoring force in the analyzed accelerometer. Using a base excitation mathematical model, the sensor output could be estimated for a specific spring constant. Although the estimated sensor output data fit well with the experimental results, the spring constant of the device could also be determined dynamically (for oscillations below $5 \mathrm{~Hz}$ ). Moreover, the damping constants could be inferred depending on whether sandblasting treatment was performed. Finally, the effects of the oscillation, surface condition, and volume of liquid metal droplets on the spring constant were analyzed.
\end{abstract}

Keywords: Liquid metal droplet, Guiding channel, Dynamic situation, Surface modification, Spring constant

\section{Introduction}

Accelerometers generally detect acceleration by the movement of the proof mass in the device [1]. Various sensing mechanisms exist for detecting the location of a proof mass (e.g., capacitive [2-6], piezoresistive [7, 8], resonant [9, 10], and optical [11, 12]). Many devices are fabricated using microelectromechanical system (MEMS) processes. Such accelerometers are composed of solid spring-like structures and can suffer from mechanical fatigue with prolonged usage [13, 14]. To improve the fatigue characteristics of solid-type MEMS accelerometers, accelerometers utilizing the properties of liquid metals (LMs) (e.g., high conductivity, density, and surface tension) have been developed [15-17].

In our recent study, a capacitive-type two-axis accelerometer using an LM was developed [17]. Because the capacitive sensing mechanism involved a dielectric structure on the electrodes, surface modification was

\footnotetext{
*Correspondence: thinking@deu.ac.kr; joonwon@postech.ac.kr ${ }^{1}$ Department of Mechanical Engineering, Pohang University of Science and Technology (POSTECH), 77 Cheongam-Ro, Nam-Gu, Pohang, Gyeongbuk 37673, South Korea

2 Division of Mechanical, Automotive and Robot Component Engineering, Dong-Eui University, 176 Eomgwang-Ro, Busanjin-Gu, Busan 47340, South Korea
}

performed on the electrode layer. In terms of the mobility of LMs in a channel, surface modifications can effectively improve the performance of the device. However, to design the device for this purpose, the spring constant should be analyzed and predicted for various situations.

In this study, the spring constant of an accelerometer using LM was analyzed. Using a base excitation mathematical model, the sensor output could be estimated for a specific spring constant. The spring constant was determined by dynamically matching the estimated values from the base excitation model and the experimental results. The damping constant was also determined using this method. Finally, the effects of the oscillation, surface condition, and volume of LM droplets on the spring constant were analyzed.

\section{Device configuration and sensing principle}

The device comprised a sensing part, an aligner, a shielding electrode (copper tape), and acrylic cases. The sensing part included a sensing printed circuit board (PCB), pressure-sensitive adhesive (PSA) film, LM, and a PMMA guiding channel. The fabrication process of the device is shown in Fig. 1a. First, a PSA film used as a dielectric layer was attached to a sensing PCB board. Subsequently, $8 \mu \mathrm{L}$ of LM droplet was deposited on the PMMA 


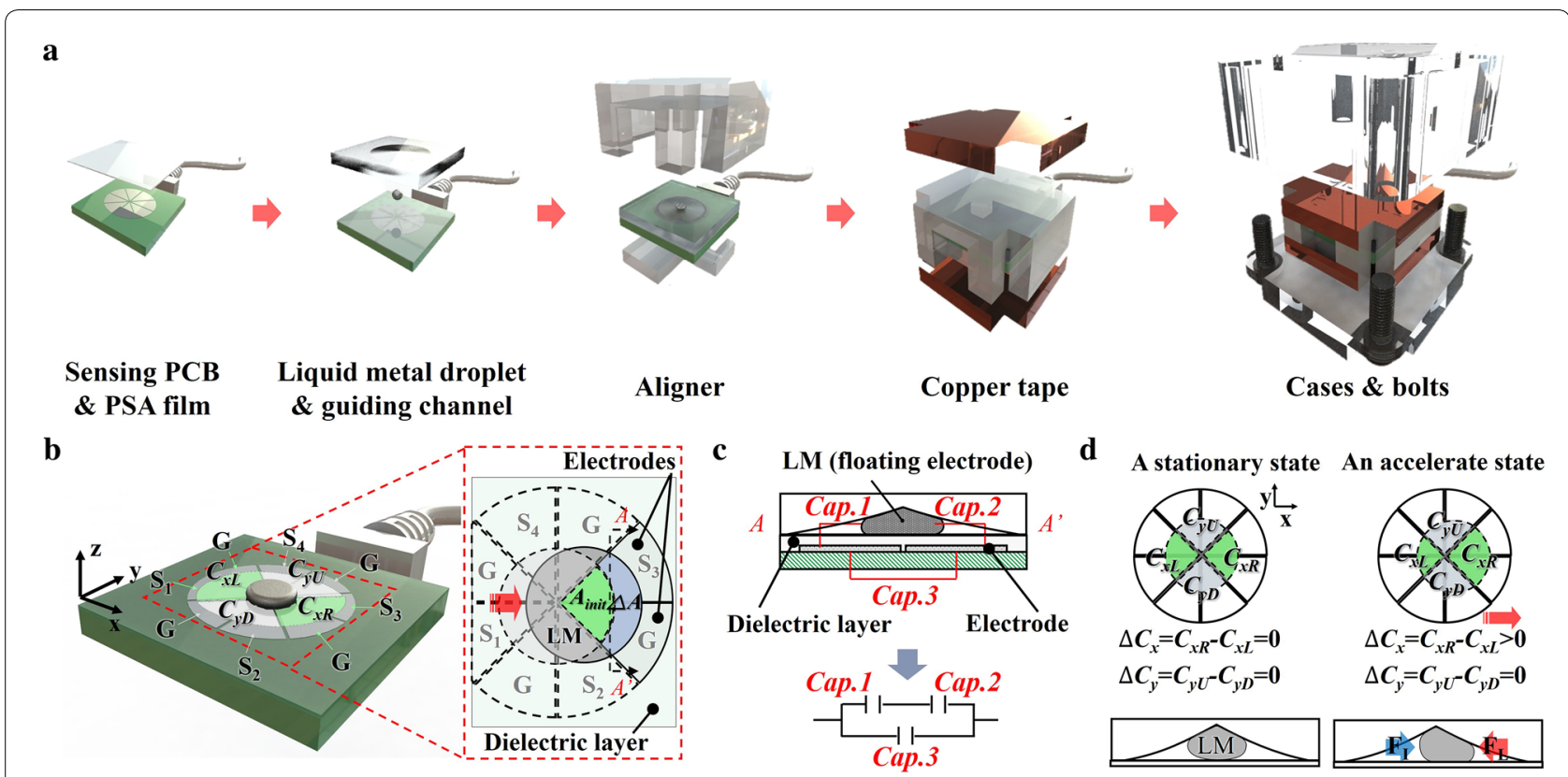

Fig. 1 Configuration and sensing principle of the device. a The fabrication process of the device. $\mathbf{b}$ Configuration of the sensing electrode. $\mathbf{c}$ Cross-sectional view of $A A^{\prime}$. Schematic diagram showing the relationship of each capacitance value (i.e., Cap. 1, Cap.2, and Cap.3). d Capacitance variations according to acceleration in the $x$-direction

guiding channel and then covered with the PCB board. Next, the channel and PCB board were fixed using the aligner. The surfaces of the PSA film and PMMA channel were applied with and without sandblasting treatment to compare the mobility of the LM droplet in the device. Sandblasting conditions of $0.3 \mathrm{MPa}$ for $10 \mathrm{~s}$ and $0.1 \mathrm{MPa}$ for $3 \mathrm{~s}$ were applied to the PMMA and PSA, respectively. The distance between the specimen and the nozzle of the sandblaster was approximately $7 \mathrm{~cm}$. Because capacitance-type sensors are easily affected by parasitic capacitance, patterned copper tape was placed on the aligner and connected to a ground for shielding. Finally, the acrylic cases were covered on both sides and fastened with M3 bolts. Using a capacitance-to-digital converter chip (AD7147, Analog Devices), each sensing cell measured $\sim 20 \mathrm{pF}$ in capacitance at $\sim 330 \mathrm{~Hz}$. The output of the counter varied linearly from 0 to 65,536 (16 bits) as the capacitance changed from approximately 4 to $25 \mathrm{pF}$.

The position of the LM was measured based on the floating electrode concept. The configuration of the sensing electrodes is shown in Fig. 1b; one set of the sensing part was paired with two cells (i.e., sensing and ground). Specifically, if the bottom cells are represented by $G$ and $S_{3}$ Cap. $_{1}$ is the capacitance generated between the LM and $G$, and $\mathrm{Cap}_{2}$ is the capacitance generated between the LM and $S_{3}$. Cap. ${ }_{3}$ represents the capacitance between the two bottom electrodes (i.e., $G$ and $S_{3}$ ), which is constant. As the area of the LM covering the two cells widened, $\mathrm{Cap}_{\cdot_{1}}$ and $\mathrm{Cap}_{\cdot_{2}}$ increased. Therefore, the overall capacitance increased as well. As shown in Fig. 1d, two sets of electrodes were arranged in the $x$ - and $y$-axis directions, separately. The acceleration of each axis was measured by the difference in capacitance at both sets of electrodes (e.g., $C_{x}=C_{x R}-C_{x L} ; C_{y}=C_{y U}-C_{y D}$ ).

When acceleration was applied, the difference in the radius of curvature across the LM droplets increased. Subsequently, the LM generated a restoring force to the center, which functioned as a spring [17]. The mobility characteristics of the LM improved owing to the surface modification on the PMMA and PSA films. This phenomenon was analyzed in terms of the damping effect (Fig. 2a).

Detailed information about the device developed in this study (e.g., characterization data and comparisons with other types of accelerometers) is provided in [17].

\section{Determination of spring constant}

\section{Spring constant and characteristic area during oscillation}

In a dynamic environment where an oscillation occurs, defining the acceleration at a specific location is difficult because the shape of the LM is not saturated. However, a sandblasting-treated device demonstrates an interesting phenomenon, wherein the LM vibrates at a natural vibration.

The setup of the oscillating device is shown in Fig. 2b. The device was attached to the end of the linear stage 


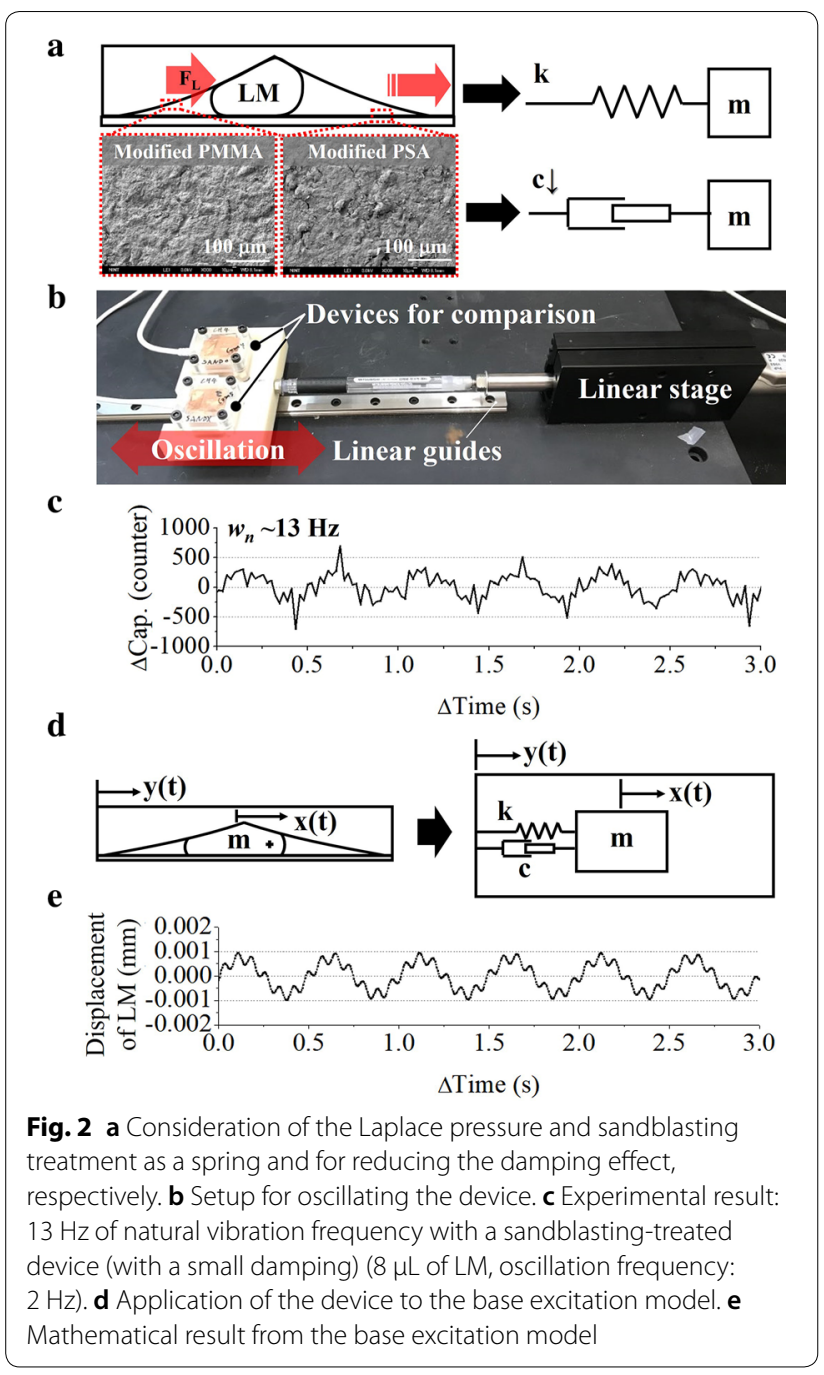

(PS01-2380-R, LinMot). To observe the behavior of an oscillating LM droplet, the copper tape for shielding was removed, and the LM vibration was recorded as a video (Additional file 1: Movie S1). An analysis of the experimental data revealed that the vibration frequency was approximately $13 \mathrm{~Hz}(8 \mu \mathrm{L}$ of LM) (Fig. 2c). To obtain the vibration frequency, the sensor output was measured using a high-pass filter to remove low-frequency signals. Based on the vibration frequency, the characteristic area in a dynamic situation can be calculated. In the case of the fabricated device, the restoring force of the proof mass is the Laplace pressure. Therefore, to obtain the spring constant, the pressure must be converted to force by multiplying it by the characteristic area. Assuming that the damping effect almost converged to zero by sandblasting treatment, the natural frequency in the spring-mass system is expressed as follows:

$$
w_{n}=\sqrt{k / m}
$$

where $w_{n}, k$, and $m$ represent the natural frequency, spring constant, and mass of the proof mass, respectively.

When $w_{n}$ was approximately $13 \mathrm{~Hz}, k$ was $0.018 \mathrm{~N} / \mathrm{m}$ for $0.10810^{-3} \mathrm{~kg}$ of $m$. Therefore, the characteristic area in the dynamic situation was approximately $0.01 \mathrm{~mm}^{2}$, such that $k$ was $0.018 \mathrm{~N} / \mathrm{m}$. Fitting can be performed by slightly adjusting the spring constant based on the spring constant for various situations (e.g., $5 \mathrm{~Hz}$ oscillation, high surface roughness, and a large volume of LM).

\section{Base excitation model}

To determine the spring and damping constants, the actual experimental result and the mathematical result obtained from the base excitation model were compared. The Laplace pressure generated from the LM droplet was described as springs. The detailed calculation of the Laplace pressure based on the location of the LM droplet is provided in [17]. Moreover, the increased mobility of the LM droplet in the sandblasting-treated device can be considered as a reduction in the damping effect. Furthermore, the base excitation mathematical model was used for validation (Fig. 2d). For specific spring and damping constants, the sensor output can be estimated using the mathematical model. When matching the estimated values calculated by the base excitation model and the experimental results in dynamic situations, the spring and damping constants can be determined. The base excitation model equation is shown in (2).

$$
m_{L M} \ddot{x}+c(\dot{x}-\dot{y})+k_{\text {Laplace }}(x-y)=0,
$$

where $m_{L M}, k_{\text {Laplace }} c, x$, and $y$ represent the mass of the LM droplet, spring constant induced by the Laplace pressure difference, damping coefficient, absolute position of the LM droplet, and absolute position of the base, respectively. The absolute position of the base with time can be expressed as (3).

$$
\mathrm{y}(t)=F_{0} \sin (w t)
$$

where $F_{0}, w$, and $t$ represent the motion amplitude, frequency of base, and time, respectively.

The result of the base excitation model is defined as $x(t) y(t)$, which is the relative position of the LM droplet at the base. The experimental setup (Fig. 2b) was established such that the reciprocating motion was exactly $y(t)$. The signals of the device were observed while the $0.03 \mathrm{~m}$ of stroke was reciprocated by two frequencies (i.e., 2 and $5 \mathrm{~Hz}$ ). The variables of the base excitation model were changed until the tendencies of the experimental result and the base excitation model were similar. 
To verify the effect of the LM droplet size, 8 and $12 \mu \mathrm{L}$ LM droplets were used for each test. $F_{0}$ was determined as $0.03 \mathrm{~m}$ in stroke length, and the LM droplet remained at the edge of the channel for a moment by inertia when acceleration was applied. At this moment, the distance from the center of the channel to the center of the LM droplet was defined as the initial position. In addition, because the initial velocity differed according to the oscillating frequency, the velocity of the LM droplet was estimated according to the frequency ratio (i.e., $0.02 \mathrm{~m} / \mathrm{s}$ at $2 \mathrm{~Hz}$ of $w$, and $0.05 \mathrm{~m} / \mathrm{s}$ at $5 \mathrm{~Hz}$ of $w$ ). The damping constant was determined by matching the experimental data with the mathematical model calculation results. The parameters of the base excitation model were determined and are listed in Table 1.

As shown in Fig. 2e, for the oscillation of an $8 \mu \mathrm{L}$ droplet at $2 \mathrm{~Hz}$, the results from the base excitation model were similar to the experimental results (Fig. 2c). A comparison of the results from the experiment and the base excitation model is shown in Fig. 3. Most of the results indicated similar tendencies; the vibration of the LM droplet reduced, and the amplitude reduced when sandblasting was not performed (the damping effect). When the sandblasting-treated device was oscillating at $5 \mathrm{~Hz}$, the LM's vibration was not suppressed as the damping effect was absent. Therefore, the vibration of the LM droplet was canceled but superimposed on the movement of the LM droplet. Consequently, the peak exhibited a random motion. A similar graph can be obtained from the mathematical model results.

\section{Reduction of spring constant}

In our previous study [17], the characteristic area in a static situation was determined based on a mathematical model and a tilting experiment. The results showed that the characteristic area in a static situation with an 8

Table 1 Parameters of the base excitation model determined according to the experimental conditions

\begin{tabular}{|c|c|c|c|c|c|c|c|c|}
\hline \multirow[b]{2}{*}{$V(\mu \mathrm{L})$} & \multicolumn{2}{|c|}{ With sandblasting } & \multicolumn{2}{|c|}{ Without sandblasting } & \multicolumn{2}{|c|}{ With sandblasting } & \multicolumn{2}{|c|}{$\begin{array}{l}\text { Without } \\
\text { sandblasting }\end{array}$} \\
\hline & 8 & & & & 12 & & & \\
\hline$m_{L M}(\mathrm{~kg})$ & 0.108 & & & & 0.162 & & & \\
\hline$F_{0}(m)$ & 0.03 & & & & & & & \\
\hline$w(\mathrm{~Hz})$ & 2 & 5 & 2 & 5 & 2 & 5 & 2 & 5 \\
\hline Initial position (m) & 0.0001 & & & & & & & \\
\hline Initial velocity $(\mathrm{m} / \mathrm{s})$ & 0.02 & 0.05 & 0.02 & 0.05 & 0.02 & 0.05 & 0.02 & 0.05 \\
\hline$k_{\text {Laplace }}(\mathrm{N} / \mathrm{m})$ & 0.018 & 0.018 & 0.016 & 0.016 & 0.023 & 0.023 & 0.021 & 0.021 \\
\hline$c(\mathrm{Ns} / \mathrm{m})$ & 0.00001 & 0.00001 & 0.0001 & 0.0001 & 0.00001 & 0.00001 & 0.0001 & 0.0001 \\
\hline
\end{tabular}

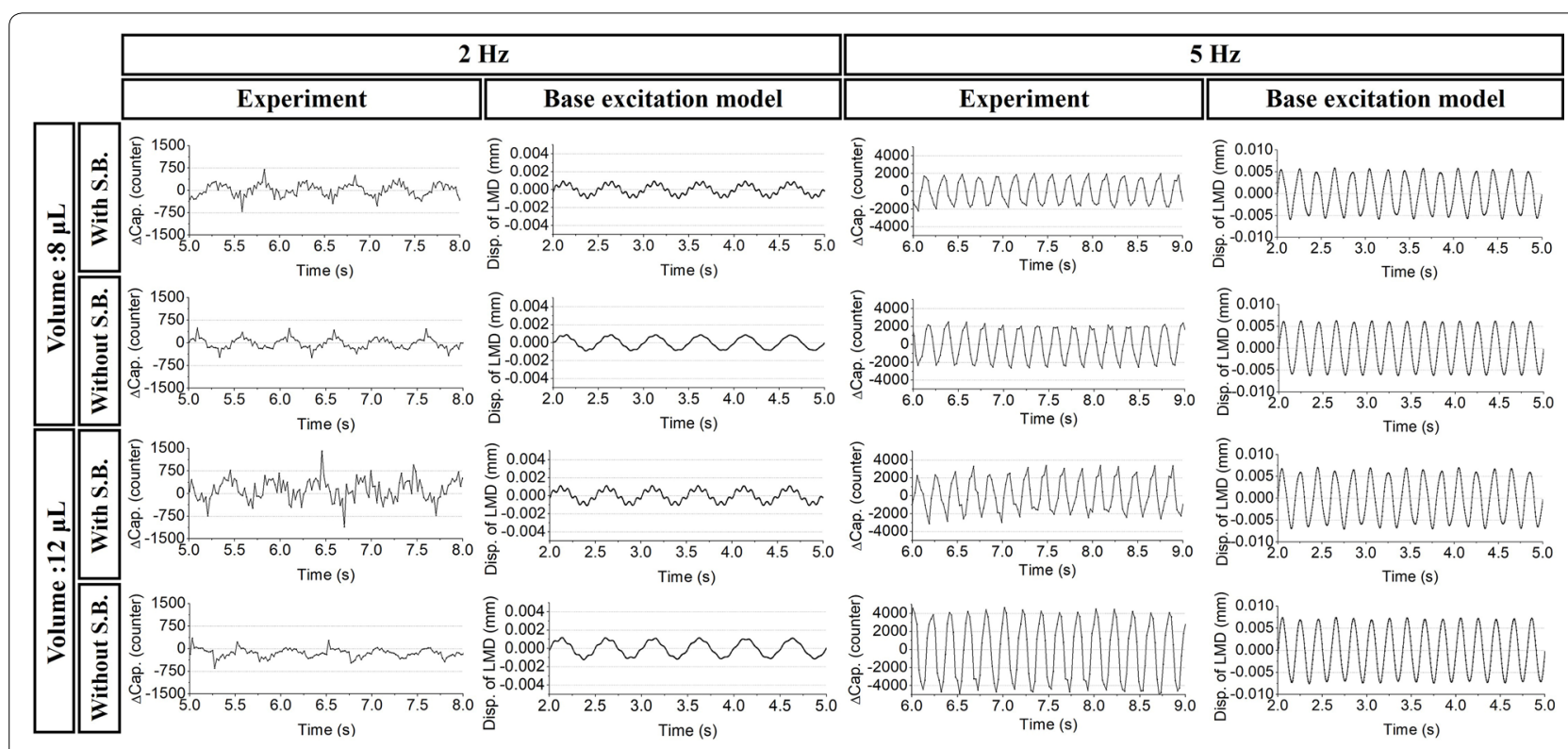

Fig. 3 Comparison of results from the experiment and base excitation mathematical model (S.B.: Sandblasting) 
$\mu \mathrm{L}$ droplet was approximately $1 \mathrm{~mm}^{2}$. As can be inferred from "Spring constant and characteristic area during oscillation" section, the characteristic area $\left(\sim 0.1 \mathrm{~mm}^{2}\right)$ in the dynamic situation was smaller than that in the static situation. The reduction in the characteristic area eventually resulted in a decrease in the spring constant. In this section, the variation in the spring constant is analyzed for various cases (i.e., oscillation, surface roughness, and volume of LM droplet).

\section{Oscillation/dynamic situation}

The characteristic area of an oscillation is less than that of a static situation (i.e., 1.0 and $0.1 \mathrm{~mm}^{2}$ for static and dynamic situations, respectively) because the time for stabilization is insufficient. For comparison, the behavior of the LM in a static situation is shown in Fig. 4a. As the LM droplet traveled, it instantaneously stretched in the direction of movement, and the curvature differences (i.e., $R_{1}$ and $R_{2}$ ) at both its ends decreased (Fig. $4 \mathrm{~b}$ ). Consequently, the Laplace pressure difference decreased, and one might predict that the spring constant would decrease in a dynamic environment.

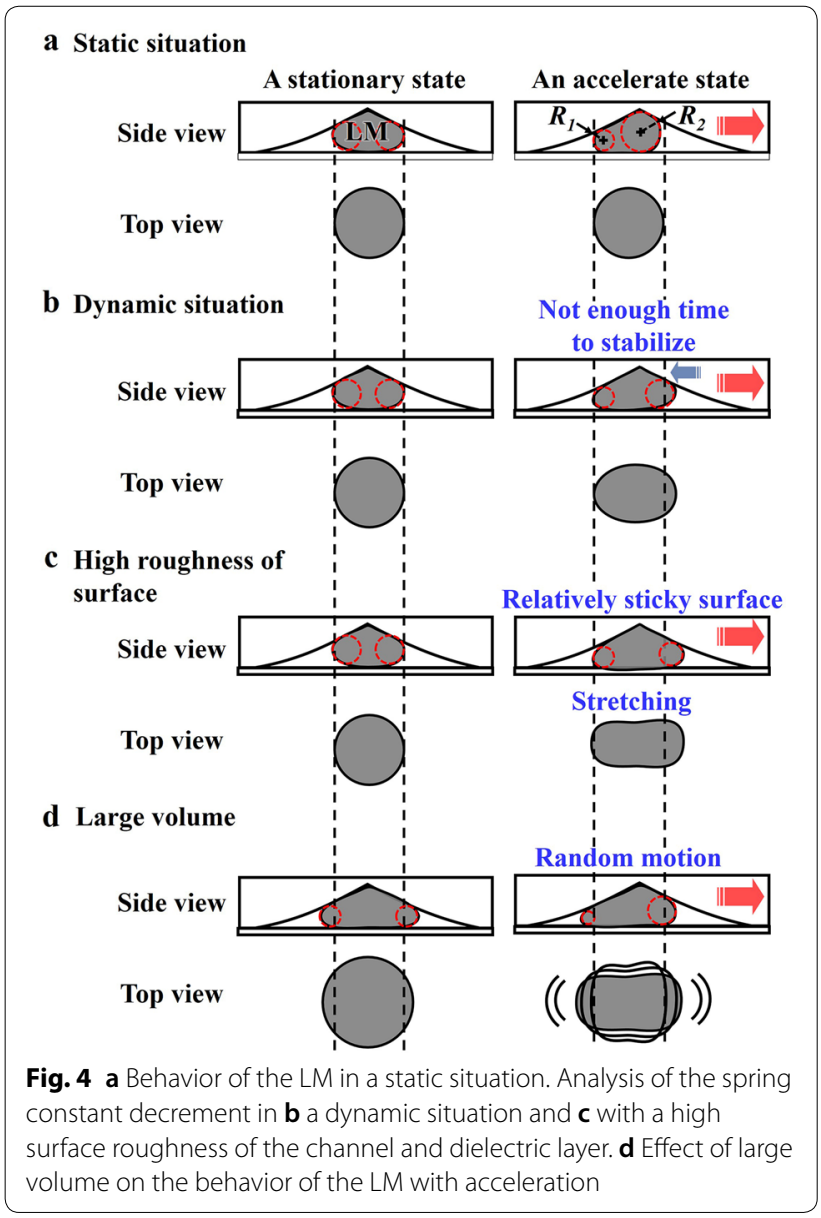

\section{Surface roughness}

The spring constant of a device with sandblasting treatment is larger than that without sandblasting treatment. The sandblasting treatment increased the surface roughness. In the sandblasting-treated device, the moving resistance of the LM droplet decreased on the upper and lower walls owing to the high surface roughness. On a surface without sandblasting treatment, the LM droplet exhibited better adhesion to the surface, and the LM stretched as it moved within the channel. Therefore, the curvature differences across the LM droplet on the surface without sandblasting treatment were smaller than those with sandblasting treatment, causing the spring constant to decrease (Fig. 4c). As shown in Fig. 3, when the device without sandblasting treatment was oscillating at $5 \mathrm{~Hz}$, although the damping constant increased, the output was larger, which supported the prediction that the device without sandblasting treatment reduced the spring constant.

\section{Effect of LM droplet volume}

As shown in Table 1, the spring constant increases slightly with LM volume. The same results were obtained from the mathematical model in [17] (Fig. 5a). As the LM volume increased, the curvature difference between both ends increased as the LM moved. Although the spring constant increased, the sensitivity of the device increased, as shown in the experimental result (Fig. 5b). Based on the results, it can be predicted that the effect of mass is more dominant than the increment in the spring constant. In Fig. 5a, $R_{3}$ represents the horizontal curvature radius of the LM (radius of

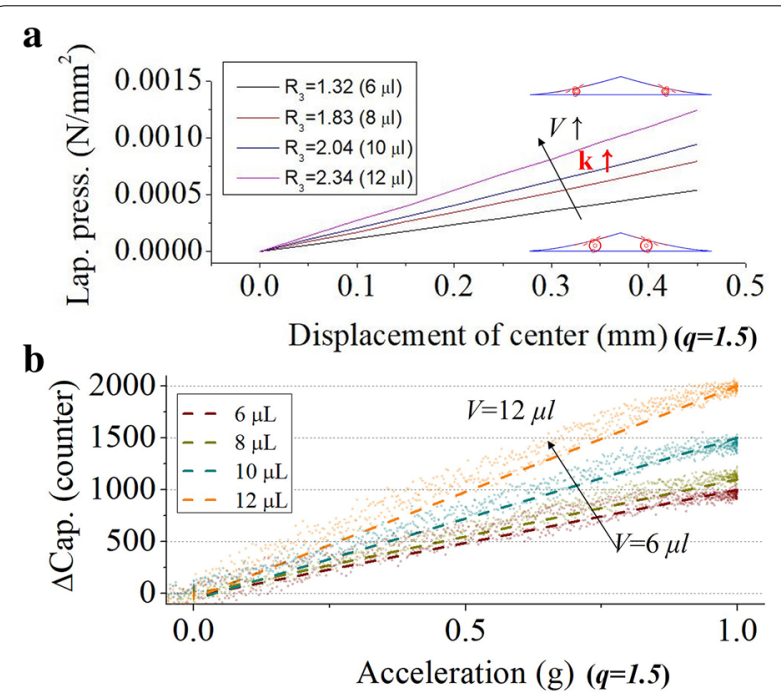

Fig. 5 Effect of the LM volume on the $\mathbf{a}$ spring constant and $\mathbf{b}$ sensitivity 
LM from the top view). Therefore, an increment in $R_{3}$ means an increment in LM volume.

Previous results indicated that an increment in volume improved the sensitivity. However, the volume should be optimized depending on the velocity range. If the LM volume is increased excessively, then the unstable behavior of the LM will increase in dynamic conditions. The instability of the LM can be explained by the Weber number (We), which defines the ratio of the kinetic energy to the surface energy, as in (4).

$$
W e=\rho L U^{2} / \sigma,
$$

where $\sigma, \rho, L$, and $U$ represent the droplet's surface tension, density, characteristic length, and velocity, respectively. In this study, $L$ and $U$ are related to the $L M$ volume and moving frequency, respectively.

If the effect of the inertia force versus the surface tension becomes large (We $\uparrow$ ), then the LM droplet cannot maintain its shape and hence exhibits various unexpected motions (e.g., oscillation-deformation, break up, etc.) [18]. These phenomena complicate the design of the accelerometer (Fig. $4 \mathrm{~d}$ ).

The volume and velocity of the LM affected the stability of our device. A large LM volume can increase sensitivity in low speed ranges but increases instability in high speed ranges. Therefore, an optimized volume that matches with the moving frequency (velocity) of the application is crucial.

\section{Conclusion}

In this paper, the spring constant in an accelerometer using a liquid-type proof mass was analyzed. The Laplace pressure induced a restoring force from a guiding channel and a LM droplet. In other words, the Laplace pressure can be considered as a spring in the device. In addition, sandblasting treatment was considered to reduce the damping effect. The outputs of the device were estimated using the base excitation mathematical model. In a dynamic situation, through an interesting phenomenon where the LM droplet vibrated at a natural vibration in a sandblasting-treated device, the characteristic area was determined. By matching the estimated outputs from the base excitation model and the oscillation experimental results, the spring and damping constants of the device were determined in a dynamic situation (for oscillations below $5 \mathrm{~Hz}$ ). The change in the spring constant of the device in a dynamic situation and the effects of high surface roughness were analyzed. Finally, the effect of the LM droplet volume on the spring constant and device sensitivity was analyzed.

\section{Supplementary information}

Supplementary information accompanies this paper at https://doi. org/10.1186/s40486-020-00115-y.

Additional file 1: Movie S1. The behavior of liquid metal droplets in the devices with (left) and without sandblasting treatments (right).

\section{Abbreviations}

LM: liquid metal; MEMS: microelectromechanical system; PCB: printed circuit board; PSA: pressure sensitive adhesive.

\section{Acknowledgements \\ Not applicable.}

\section{Authors' contributions}

DJW carried out the experiments and drafted the manuscript. SL analyzed the experimental result. JK drafted the manuscript. All authors read and approved the final manuscript.

\section{Funding}

This work was supported by the National Research Foundation of Korea (NRF) Grant funded by the Korea government (MSIT) (No. 2017R1C1B5076710).

\section{Availability of data and materials}

The datasets and a movie supporting the conclusions of this article are included within the article.

\section{Competing interests}

The authors declare that they have no competing interests.

Received: 27 April 2020 Accepted: 14 July 2020

Published online: 22 July 2020

\section{References}

1. Shaeffer DK (2013) MEMS inertia sensors: a tutorial overview. IEEE Commun Mag 51(4):100-109. https://doi.org/10.1109/MCOM.2013.6495768

2. Li Q (2015) Fabrication of a high sensitivity MEMS accelerometer with symmetrical double-sided serpentine beam-mass structure. In: 2015 IEEE SENSORS, Busan, South Korea, 1-4 November 2015. https://doi. org/10.1109/icsens.2015.7370430

3. Zhou X (2015) Design and fabrication of a MEMS capacitive accelerometer with fully symmetrical double-sided $\mathrm{H}$-shaped beam structure. Microelectron Eng 131:51-57. https://doi.org/10.1016/j.mee.2014.10.005

4. Sun CM (2008) On the sensitivity improvement of CMOS capacitive accelerometer. Sens Actuator A-Phys 141(2):347-352. https://doi.org/10.1016/j. sna.2007.10.026

5. Gomathi T (2016) Capacitive accelerometers for microelectromechanical applications: a review. In: 2016 ICCICCT, Kumaracoil, India, 16-17 December 2016. https://doi.org/10.1109/iccicct.2016.7987999

6. Mohammed Z (2018) Monolithic multi degree of freedom (MDoF) capacitive MEMS accelerometers. Micromachines 9(11):602. https://doi. org/10.3390/mi9110602

7. Partridge A (2000) A high-performance planar piezoresistive accelerometer. J Microelectromech Syst 9(1):58-66. https://doi.org/10.1109/84.82577 8

8. Hsieh HS (2011) A novel stress isolation guard-ring design for the improvement of a three-axis piezoresistive accelerometer. In: 2011 16th International Solid-State Sensors, Actuators and Microsystems Conference, Beijing, China, 5-9 June 2011. https://doi.org/10.1109/transducer s.2011.5969582

9. Park U (2014) A micromachined differential resonant accelerometer based on robust structural design. Microelectron Eng 129:5-11. https:// doi.org/10.1016/j.mee.2014.06.008

10. Zhang J (2015) Microelectromechanical resonant accelerometer designed with a high sensitivity. Sensors 15(12):30293-30310. https://doi. org/10.3390/s151229803 
11. Krause AG (2012) A high-resolution microchip optomechanical accelerometer. Nat Photonics 6:768-772. https://doi.org/10.1038/nphot on.2012.245

12. Cervantes FG (2014) High sensitivity optomechanical reference accelerometer over 10 kHz. Appl Phys Lett 104(22):221111. https://doi. org/10.1063/1.4881936

13. Langfelder $G$ (2012) The dependence of fatigue in microelectromechanical systems on the environment and the industrial packaging. IEEE Trans Ind Electron 59(12):4938-4948. https://doi.org/10.1109/TIE.2011.2151824

14. Mariani S (2008) A three-scale FE approach to reliability analysis of MEMS sensors subject to impacts. Meccanica 43(5):469-483. https://doi. org/10.1007/s11012-008-9111-0

15. Park U (2010) Development of a MEMS digital accelerometer (MDA) using a microscale liquid metal droplet in a microstructured photosensitive glass channel. Sens Actuator A-Phys 159(1):51-57. https://doi. org/10.1016/j.sna.2010.02.011
16. Huh M (2017) Simple and robust resistive dual-axis accelerometer using a liquid metal droplet. Micro Nano Syst Lett 5:5. https://doi.org/10.1186/ s40486-016-0038-2

17. Won D (2020) Capacitive-type two-axis accelerometer with liquid-type proof mass. Adv Electron Mater. https://doi.org/10.1002/aelm.201901265

18. Chen Y (2017) Aerodynamic breakup and secondary drop formation for a liquid metal column in a shock-induced cross-flow. In: 55th AIAA Aerospace Sciences Meeting, AIAA, Grapevine, United States, 9-13 January 2017. https://doi.org/10.2514/6.2017-1892

\section{Publisher's Note}

Springer Nature remains neutral with regard to jurisdictional claims in published maps and institutional affiliations.

\section{Submit your manuscript to a SpringerOpen ${ }^{\circ}$ journal and benefit from:}

- Convenient online submission

- Rigorous peer review

- Open access: articles freely available online

- High visibility within the field

- Retaining the copyright to your article

Submit your next manuscript at $\gg$ springeropen.com 